\title{
THE SET OF SOLUTIONS OF INTEGRODIFFERENTIAL EQUATIONS IN BANACH SPACES
}

\author{
RAVI P. AGARWAL, DONAL O'REGAN and ANETA SIKORSKA-NOWAK
}

\author{
(Received 17 March 2008)
}

\section{Abstract}

In this paper, we first prove an existence theorem for the integrodifferential equation

$$
\left\{\begin{array}{l}
x^{\prime}(t)=f\left(t, x(t), \int_{0}^{t} k(t, s, x(s)) d s\right), \quad t \in I_{a}=[0, a], a \in R_{+}, \\
x(0)=x_{0}
\end{array}\right.
$$

where $f, k, x$ are functions with values in a Banach space $E$ and the integral is taken in the sense of Henstock-Kurzweil-Pettis. In the second part of the paper we show that the set $S$ of all solutions of the problem $(*)$ is compact and connected in $\left(C\left(I_{d}, E\right), \omega\right)$, where $I_{d} \subset I_{a}$.

2000 Mathematics subject classification: 34D09, 34D99.

Keywords and phrases: integral equations, existence theorem, pseudo-solution, set of solutions, measure of noncompactness, Henstock-Kurzweil-Pettis integral.

\section{Introduction}

In this paper, we prove an existence theorem for the problem

$$
\left\{\begin{array}{l}
x^{\prime}(t)=f\left(t, x(t), \int_{0}^{t} k(t, s, x(s)) d s\right), \quad t \in I_{a}=[0, a], a \in R_{+}, x_{0} \in E, \\
x(0)=x_{0},
\end{array}\right.
$$

where $f, k, x$ are functions with values in a Banach space $E$ and the integral is taken in the sense of Henstock-Kurzweil-Pettis [13].

The Henstock-Kurzweil integral encompasses the Newton, Riemann and Lebesgue integrals [17, 19, 24]. A particular feature of this integral is that the integral of highly oscillating functions such as $F^{\prime}(t)$, where $F(t)=t^{2} \sin t^{-2}$ on $(0,1]$ and $F(0)=0$, can be defined. This integral was introduced by Henstock and Kurzweil independently in 1957-58 and has since proved useful in the study of ordinary

(c) 2009 Australian Mathematical Society 0004-9727/09 \$A2.00+0.00 
differential equations [4, 8, 9, 23]. In [12] the authors defined the Henstock-KurzweilPettis integral, which is a generalization of the Henstock-Kurzweil integral and the Pettis integral.

The existence theorem presented in this paper is an extension of previous results, for example [1-3, 14, 20, 21, 25, 27, 28].

Let $(E,\|\cdot\|)$ be a Banach space and let $E^{*}$ be the dual space. Moreover, let $\left(C\left(I_{a}, E\right), \omega\right)$ denote the space of all continuous functions from $I_{a}$ to $E$ endowed with the topology $\sigma\left(C\left(I_{a}, E\right), C\left(I_{a}, E\right)^{*}\right)$.

In this paper we prove that the set $S$ of all solutions of the integrodifferential equation (1.1) on $I_{d}=[0, d], 0<d \leq a$, is connected and compact in $\left(C\left(I_{d}, E\right), \omega\right)$. This problem was investigated by Cichoń and Kubiaczyk [11, 22], Szufla [30] and others.

Let us recall, that a function $f: I_{a} \rightarrow E$ is said to be weakly continuous if it is continuous from $I_{a}$ to $E$ endowed with its weak topology. A function $g: E \rightarrow E_{1}$, where $E$ and $E_{1}$ are Banach spaces, is said to be weakly-weakly sequentially continuous if for each weakly convergent sequence $\left(x_{n}\right) \subset E$, the sequence $\left(g\left(x_{n}\right)\right)$ is weakly convergent in $E_{1}$. If a sequence $x_{n}$ tends weakly to $x_{0}$ in $E$ we denote it by $x_{n} \rightarrow{ }^{\omega} x_{0}$.

The fundamental tool in this paper is the measure of weak noncompactness developed by DeBlasi [6] and Banaś and Rivero [5].

Let $A$ be a bounded nonempty subset of $E$.

The measure of weak noncompactness $\beta(A)$ is defined by

$$
\beta(A)=\inf \left\{t>0 \mid \text { there exists } C \in K^{\omega} \text { such that } A \subset C+t B_{0}\right\},
$$

where $K^{\omega}$ is a set of weakly compact subsets of $E$ and $B_{0}$ is a norm unit ball in $E$. Some properties of the measure of weak noncompactness $\beta(A)$ are:

(i) if $A \subset B$, then $\beta(A) \leq \beta(B)$;

(ii) $\beta(A)=\beta\left(\overline{A^{w}}\right)$, where $\overline{A^{w}}$ denotes the weak closure of $A$;

(iii) $\beta(A)=0$ if and only if $A$ is relatively weakly compact;

(iv) $\beta(A \cup B)=\max \{\beta(A), \beta(B)\}$;

(v) $\beta(\lambda A)=|\lambda| \beta(A)(\lambda \in R)$;

(vi) $\beta(A+B) \leq \beta(A)+\beta(B)$;

(vii) $\beta(\overline{\operatorname{conv}}(A))=\beta(\operatorname{conv}(A))=\beta(A)$, where $\operatorname{conv}(A)$ denotes the convex hull of $A$.

LEMMA 1.1 [26]. Let $H \subset C\left(I_{a}, E\right)$ be a family of strongly equicontinuous functions. Let for $t \in I_{a}, H(t)=\{h(t) \in E, h \in H\}$. Then $\beta\left(H\left(I_{a}\right)\right)=\sup _{t \in I_{a}} \beta(H(t))$ and the function $t \rightarrow \beta(H(t))$ is continuous.

LeMma 1.2 [11]. Let $(X, d)$ be a metric space and let $f: X \rightarrow(E, \omega)$ be sequentially continuous. If $A \subset X$ is a connected subset in $X$, then $f(A)$ is the connected subset in $(E, \omega)$. 
Fix $x^{*} \in E^{*}$ and consider the problem

$$
\left\{\begin{array}{l}
\left(x^{*} x\right)^{\prime}=x^{*}\left(f\left(t, x(t), \int_{0}^{t} k(t, s, x(s)) d s\right)\right), \quad t \in I_{a}, x_{0} \in E . \\
x(0)=x_{0},
\end{array}\right.
$$

Let us introduce the following definitions.

Definition 1.3 [29]. Let $F:[a, b] \rightarrow E$ and let $A \subset[a, b]$. The function $f: A \rightarrow E$ is a pseudoderivative of $F$ on $A$ if for each $x^{*} \in E^{*}$ the real-valued function $x^{*} F$ is differentiable almost everywhere on $A$.

It is clear that the left-hand side of (1.2) can be rewritten in the form $x^{*}\left(x^{\prime}(t)\right)$ where $x^{\prime}$ denotes the pseudoderivative.

Definition $1.4[17,24]$. A family $\mathbf{F}$ of functions $F$ is said to be uniformly absolutely continuous in the restricted sense on $A$ (or uniformly $A C^{*}(A)$ for short), if for every $\varepsilon>0$ there is $\eta>0$ such that for every $F$ in $\mathbf{F}$ and for every finite or infinite sequence of nonoverlapping intervals $\left\{\left[a_{i}, b_{i}\right]\right\}$ with $a_{i}, b_{i} \in A$ and satisfying $\sum_{i}\left|b_{i}-a_{i}\right|<\eta$, we have $\sum_{i} \omega\left(F,\left[a_{i}, b_{i}\right]\right)<\varepsilon$ where $\omega\left(F,\left[a_{i}, b_{i}\right]\right)$ denotes the oscillation of $F$ over $\left[a_{i}, b_{i}\right]$ (that is, $\omega\left(F,\left[a_{i}, b_{i}\right]=\sup \left\{|F(r)-F(s)|: r, s \in\left[a_{i}, b_{i}\right]\right\}\right)$. A family $\mathbf{F}$ of functions $F$ is said to be uniformly generalized absolutely continuous in the restricted sense on $[a, b]$ or uniformly $A C G^{*}$ on $[a, b]$ if $[a, b]$ is the union of a sequence of closed sets $A_{i}$ such that on each $A_{i}$, the family $\mathbf{F}$ is uniformly $A C^{*}\left(A_{i}\right)$.

\section{Henstock-Kurzweil-Pettis integrals in Banach spaces}

In this section we present a definition of the Henstock-Kurzweil-Pettis integral, which is a generalization of both Pettis and Henstock-Kurzweil integrals. For basic definitions we refer the reader to [17] or [24].

Definition 2.1 $[17,24]$. Let $\delta$ be a positive function defined on the interval $[a, b]$. A tagged interval $(x,[c, d])$ consists of an interval $[c, d] \subseteq[a, b]$ and a point $x \in[c, d]$. The tagged interval $(x,[c, d])$ is subordinate to $\delta$ if $[c, d] \subseteq(x-\delta(x), x+\delta(x))$.

Let $P=\left\{\left(s_{i},\left[c_{i}, d_{i}\right]\right) \mid 1 \leq i \leq n, n \in N\right\}$ be such a collection in $[a, b]$. Then:

(i) the points $\left\{s_{i} \mid 1 \leq i \leq n\right\}$ are called the tags of $P$;

(ii) the intervals $\left\{\left[c_{i}, d_{i}\right] \mid 1 \leq i \leq n\right\}$ are called the intervals of $P$;

(iii) if $\left\{\left(s_{i},\left[c_{i}, d_{i}\right]\right) \mid 1 \leq i \leq n\right\}$ is subordinate to $\delta$ for each $i$, then we write $P$ is sub- $\delta$;

(iv) if $[a, b]=\bigcup_{i=1}^{n}\left[c_{i}, d_{i}\right]$, then $P$ is called a tagged partition of $[a, b]$;

(v) if $P$ is a tagged partition of $[a, b]$ and if $P$ is sub- $\delta$, then we write $P$ is $s u b-\delta$ on $[a, b]$;

(vi) if $f:[a, b] \rightarrow E$, then $f(P)=\sum_{i=1}^{n} f\left(s_{i}\right)\left(d_{i}-c_{i}\right)$;

(vii) if $F$ is defined on the subintervals of $[a, b]$, then

$$
F(P)=\sum_{i=1}^{n} F\left(\left[c_{i}, d_{i}\right]\right)=\sum_{i=1}^{n}\left[F\left(d_{i}\right)-F\left(c_{i}\right)\right] .
$$


If $F:[a, b] \rightarrow E$, then $F$ can be treated as a function of intervals by defining $F([c, d])=F(d)-F(c)$. For such a function, $F(P)=F(b)-F(a)$ if $P$ is a tagged partition of $[a, b]$.

Definition $2.2[17,24]$. A function $f:[a, b] \rightarrow R$ is Henstock-Kurzweil integrable on $[a, b]$ if there exists a real number $L$ with the following property: for each $\varepsilon>0$ there exists a positive function $\delta$ on $[a, b]$ such that $|f(P)-L|<\varepsilon$ whenever $P$ is a tagged partition of $[a, b]$ that is subordinate to $\delta$.

The function $f$ is Henstock-Kurzweil integrable on a measurable set $A \subset[a, b]$ if $f \chi_{A}$ is Henstock-Kurzweil integrable on $[a, b]$. The number $L$ is called the HenstockKurzweil integral of $f$. We denote this integral by $(H K) \int_{a}^{b} f(t) d t$.

Definition 2.3 [7]. A function $f:[a, b] \rightarrow E$ is Henstock-Kurzweil integrable on $[a, b](f \in H K([a, b], E))$ if there exists a vector $z \in E$ with the following property: for every $\varepsilon>0$ there exists a positive function $\delta$ on $[a, b]$ such that $\|f(P)-z\|<\varepsilon$ whenever $P$ is a tagged partition of $[a, b]$ sub- $\delta$. The function $f$ is Henstock-Kurzweil integrable on a measurable set $A \subset[a, b]$ if $f \chi_{A}$ is Henstock-Kurzweil integrable on $[a, b]$. The vector $z$ is the Henstock-Kurzweil integral of $f$.

Note that this definition includes the generalized Riemann integral defined by Gordon [18].

Definition 2.4 [7]. A function $f:[a, b] \rightarrow E$ is $H L$ integrable on $[a, b]$ $(f \in H L([a, b], E))$ if there exists a function $F:[a, b] \rightarrow E$, defined on the subintervals of $[a, b]$, satisfying the following property: given $\varepsilon>0$ there exists a positive function $\delta$ on $[a, b]$ such that if $P=\left\{\left(s_{i},\left[c_{i}, d_{i}\right] \mid 1 \leq i \leq n\right\}\right.$ is a tagged partition of $[a, b]$ sub- $\delta$, then

$$
\sum_{i=1}^{n}\left\|f\left(s_{i}\right)\left(d_{i}-c_{i}\right)-F\left(\left[c_{i}, d_{i}\right]\right)\right\|<\varepsilon
$$

REMARK 2.5. We note that by the triangle inequality:

$$
f \in H L([a, b], E) \quad \text { implies } f \in H K([a, b], E) .
$$

In general, the converse is not true. For real-valued functions, the two integrals are equivalent.

Definition 2.6 [29]. The function $f: I_{a} \rightarrow E$ is Pettis integrable (P integrable for short) if:

(i) for all $x^{*} \in E^{*}, x^{*} f$ is Lebesgue integrable on $I_{\alpha}$; and

(ii) for all $A \subset I_{a}, A$-measurable, there exists $g \in E$ for all $x^{*} \in E^{*}$ such that $x^{*} g=(L) \int_{A} x^{*} f(s) d s$.

Now we present a definition of the integral which is a generalization both Pettis and Henstock-Kurzweil integrals. 
Definition 2.7 [13]. The function $f: I_{a} \rightarrow E$ is Henstock-Kurzweil-Pettis integrable (HKP integrable for short) if there exists a function $g: I_{a} \rightarrow E$ with the following properties:

(i) for all $x^{*} \in E^{*}, x^{*} f$ is Henstock-Kurzweil integrable on $I_{a}$; and

(ii) for all $t \in I_{a}$ and all $x^{*} \in E^{*}, x^{*} g(t)=(H K) \int_{0}^{t} x^{*} f(s) d s$.

This function $g$ is called a primitive of $f$ and by $g(a)=\int_{0}^{a} f(t) d t$ we denote the Henstock-Kurzweil-Pettis integral of $f$ on the interval $I_{a}$.

REMARK 2.8. Each function which is HL integrable is integrable in the sense of Henstock-Kurzweil-Pettis. This notion of an integral is essentially more general than previous notions (in Banach spaces).

(i) Pettis integral: by the definition of the Pettis integral and since each Lebesgue integrable function is $\mathrm{HK}$ integrable, a $\mathrm{P}$ integrable function is clearly HKP integrable;

(ii) Bochner, Riemann, and Riemann-Pettis integrals [18];

(iii) MsShane integral [16];

(iv) Henstock-Kurzweil (HL) integral [7].

We present below an example of function which is HKP integrable but neither HL integrable nor $\mathrm{P}$ integrable.

EXAmple. Let $f:[0,1] \rightarrow\left(L^{\infty}[0,1],\|\cdot\|_{\infty}\right)$ and let $f(t)=\chi_{[0, t]}+A(t) \cdot F^{\prime}(t)$, where

$$
F(t)=t^{2} \sin t^{-2}, \quad t \in(0,1], \quad F(0)=0, \quad \chi_{[0, t]}(\tau)=\left\{\begin{array}{l}
1, \tau \in[0, t], \\
0, \tau \notin[0, t],
\end{array} \quad t, \tau \in[0,1],\right.
$$

and $A(t)(\tau)=1$ for $\tau, t \in[0,1]$.

Put $f_{1}(t)=\chi_{[0, t]}, f_{2}(t)=A(t) \cdot F^{\prime}(t)$.

We show that a function $f(t)=f_{1}(t)+f_{2}(t)$ is integrable in the sense of Henstock-Kurzweil-Pettis.

Observe that

$$
x^{*}(f(t))=x^{*}\left(f_{1}(t)+f_{2}(t)\right)=x^{*}\left(f_{1}(t)\right)+x^{*}\left(f_{2}(t)\right) .
$$

Moreover, the function $x^{*}\left(f_{1}(t)\right)$ is Lebesgue integrable (in fact $f_{1}$ is $\mathrm{P}$ integrable [15], so is Henstock-Kurzweil integrable, and the function $x^{*}\left(f_{2}(t)\right)$ is Henstock-Kurzweil integrable by Definition 2.2.

For each $x^{*} \in E^{*}$ the function $x^{*} f$ is not Lebesgue integrable because $x^{*} f_{2}$ is not Lebesgue integrable. Thus, $f$ is not $\mathrm{P}$ integrable. Moreover, the function $f_{1}$ is not strongly measurable [15] and the function $f_{2}$ is strongly measurable. Thus, their sum $f$ is not strongly measurable. Then, by [7, Theorem 9], $f$ is not HL integrable.

Now we list some properties of the HKP integral which are important in the next sections of our paper. 
THEOREM 2.9. Let $f:[a, b] \rightarrow E$ be HKP integrable on $[a, b]$ and let

$$
F(x)=\int_{a}^{x} f(s) d s, \quad x \in[a, b] .
$$

Then:

(i) for each $x^{*}$ in $E^{*}$ the function $x^{*} f$ is HK integrable on $[a, b]$ and

$$
(H K) \int_{a}^{x} x^{*}(f(s)) d s=x^{*}(F(x)) ;
$$

(ii) the function $F$ is weakly continuous on $[a, b]$ and $f$ is a pseudoderivative of $F$ on $[a, b]$.

THEOREM 2.10 [13]. Let $f:[a, b] \rightarrow E$. If $f=0$ almost everywhere on $[a, b]$, then $f$ is HKP integrable on $[a, b]$ and $\int_{a}^{b} f(t) d t=0$.

Theorem 2.11 ([13] Mean value theorem for the HKP integral). If the function $f: I_{a} \rightarrow E$ is HKP integrable, then

$$
\int_{I} f(t) d t \in|I| \cdot \overline{\operatorname{conv}} f(I)
$$

where $I$ is an arbitrary subinterval of $I_{a}$ and $|I|$ is the length of $I$.

TheOREM 2.12 [10]. Let $f: I_{a} \rightarrow E$ and assume that $f_{n}: I_{a} \rightarrow E, n \in N$ are HKP integrable on $I_{a}$. Let $F_{n}$ be a primitive of $f_{n}$. If we assume that:

(i) for all $x^{*} \in E^{*}, x^{*}\left(f_{n}(t)\right) \rightarrow x^{*}(f(t))$ almost everywhere on $I_{a}$;

(ii) for each $x^{*} \in E^{*}$ the family $G=\left\{x^{*} F_{n} \mid n=1,2, \ldots\right\}$ is uniformly $A C G^{*}$ on $I_{a}$ (that is, weakly uniformly $A C G^{*}$ on $I_{a}$ );

(iii) for each $x^{*} \in E^{*}$ the set $G$ is equicontinuous on $I_{a}$;

then $f$ is HKP integrable on $I_{a}$ and $\int_{0}^{t} f_{n}(s) d s$ tends weakly in $E$ to $\int_{0}^{t} f(s) d s$ for each $t \in I_{a}$.

\section{An existence result for integrodifferential equations in the weak sense}

In this section, we prove an existence theorem for problem (1.1).

Let

$$
\begin{aligned}
& B=\left\{x \in E:\|x\| \leq\left\|x_{0}\right\|+b, b>0\right\} \\
& \widetilde{B}=\left\{x \in\left(C\left(I_{a}, E\right), \omega\right): x(0)=x_{0},\|x\| \leq\left\|x_{0}\right\|+b, b>0\right\} .
\end{aligned}
$$


Moreover, let

$$
\begin{aligned}
F(x)(t) & =x_{0}+\int_{0}^{t} f\left(z, x(z), \int_{0}^{z} k(z, s, x(s)) d s\right) d z, t \in I_{a}, \\
K & =\{F(x) \mid x \in \widetilde{B}\}, \\
K_{1} & =\left\{\int_{0}^{z} k(z, s, x(s)) d s \mid z \in[0, t], t \in[0, a], x \in \widetilde{B}\right\} .
\end{aligned}
$$

We consider the problem

$$
x(t)=x_{0}+\int_{0}^{t} f\left(z, x(z), \int_{0}^{z} k(z, s, x(s)) d s\right) d z, \quad t \in I_{a},
$$

where the integrals are taken in the sense of HKP.

To obtain the existence result and to investigate the structure of a solution set for our problem it is necessary to define the notion of a solution.

Definition 3.1 [29]. A function $x: I_{a} \rightarrow E$ is said to be a pseudo-solution of the problem (1) if it satisfies the following conditions:

(i) $x(\cdot)$ is $A C G_{*}$ function;

(ii) $x(0)=x_{0}$;

(iii) for each $x^{*} \in E^{*}$ there exists a set $A\left(x^{*}\right)$ with Lebesgue measure zero, such that for each $t \notin A\left(x^{*}\right)$,

$$
\left(x^{*} x\right)^{\prime}(t)=x^{*}\left(f\left(t, x(t), \int_{0}^{t} k(t, s, x(s)) d s\right)\right) .
$$

Here / denotes the pseudoderivative.

In the proof of the main theorem we shall apply the following result.

THEOREM 3.2 [22]. Let $E$ be a metrizable locally convex topological vector space. Let $D$ be a closed convex subset of $E$, and let $F$ be a weakly-weakly sequentially continuous map of $D$ into itself. If, for some $x \in D$, the implication

$$
\bar{V}=\overline{\operatorname{conv}}(\{x\} \cup F(V)) \text { implies } V \text { is relatively weakly compact, }
$$

holds for every subset $V$ of $D$, then $F$ has a fixed point.

THEOREM 3.3. Assume that for each uniformly $A C G^{*}$ function $x: I_{a} \rightarrow E$, the functions $k(\cdot, s, x(s)), \quad f\left(\cdot, x(\cdot), \int_{0}^{(\cdot)} k(\cdot, s, x(s)) d s\right)$ are HKP integrable and $k(t, s, \cdot), f(t, \cdot, \cdot)$ are weakly-weakly sequentially continuous functions. Suppose that there exists constants $c_{1}, c_{2}, c_{3}>0$ such that

$$
\begin{aligned}
& \beta(f(I, A, C)) \leq c_{1} \cdot \beta(A)+c_{2} \cdot \beta(C) \text { for each } A, C \subset B, I \subset I_{a}, \\
& \beta(k(I, I, X)) \leq c_{3} \cdot \beta(X) \text { for each } X \subset B, I \subset I_{a},
\end{aligned}
$$


where

$$
\begin{aligned}
f(I, A, C) & =\left\{f\left(t, x_{1}, x_{2}\right) \mid\left(t, x_{1}, x_{2}\right) \in I \times A \times C\right\}, \\
k(I, I, X) & =\{k(t, s, x) \mid(t, s, x) \in I \times I \times X\}
\end{aligned}
$$

and $\beta$ denotes the measure of weak noncompactness of DeBlasi.

Moreover, let $K$ and $K_{1}$ be equicontinuous and uniformly $A C G^{*}$ on $I_{a}$. Then there exists a pseudo-solution of the problem (1.1) on $I_{d}$, for some $0<d \leq a$, $0<d \cdot c_{1}+d^{2} \cdot c_{2} \cdot c_{3}<1$.

PROOF. Fix an arbitrary $b \geq 0$. Put

$$
\begin{aligned}
& B=\left\{x \in E:\|x\| \leq\left\|x_{0}\right\|+b, b>0\right\}, \\
& \widetilde{B}=\left\{x \in\left(C\left(I_{d}, E\right), \omega\right): x(0)=x_{0},\|x\| \leq\left\|x_{0}\right\|+b, b>0\right\},
\end{aligned}
$$

where $d$ is given below.

Recall that a continuous function $F(x) \in K$ defined on $[0, a]$ is equicontinuous on $[0, a]$, if for each $\varepsilon>0$ there exists $\delta>0$ such that $\|F(x)(t)-F(x)(\tau)\|<\varepsilon$, for all $x \in \widetilde{B}$, whenever $|t-\tau|<\delta$ and $t, \tau \in[0, a]$. Thus, for each $\varepsilon>0$ there exists $\delta>0$, such that

$$
\left\|\int_{\tau}^{t} f\left(z, x(z), \int_{0}^{z} k(z, s, x(s)) d s\right) d z\right\|<\varepsilon
$$

for all $x \in \widetilde{B}$, whenever $|t-\tau|<\delta$ and $t, \tau \in[0, a]$. As a result, there exists a number $d, 0<d \leq a$, such that

$$
\left\|\int_{0}^{t} f\left(z, x(z), \int_{0}^{z} k(z, s, x(s)) d s\right) d z\right\| \leq b, \quad t \in I_{d} \text { and } x \in \widetilde{B} .
$$

We now show that the operator $F$ is well defined and maps $\widetilde{B}$ into $\widetilde{B}$. To see this note that, for any $x^{*} \in E^{*}$, such that $\left\|x^{*}\right\| \leq 1$, for any $x \in \widetilde{B}$ and $t \in I_{d}$

$$
\begin{aligned}
\left|x^{*} F(x)(t)\right| & \leq\left|x^{*} x_{0}\right|+\left|x^{*} \int_{0}^{t} f\left(z, x(z), \int_{0}^{z} k(z, s, x(s)) d s\right) d z\right| \\
& \leq\left\|x^{*}\right\|\left\|x_{0}\right\|+\left\|x^{*}\right\|\left\|\int_{0}^{t} f\left(z, x(z), \int_{0}^{z} k(z, s, x(s)) d s\right) d z\right\| \\
& \leq\left\|x_{0}\right\|+b,
\end{aligned}
$$

so

$$
\sup \left\{\left|x^{*} F(x)(t)\right|: x^{*} \in E^{*},\left\|x^{*}\right\| \leq 1\right\} \leq\left\|x_{0}\right\|+b
$$

and as a result

$$
\|F(x)(t)\| \leq\left\|x_{0}\right\|+b,
$$

so $F(x)(t) \in \widetilde{B}$.

We shall show that the operator $F$ is weakly-weakly sequentially continuous. By [26, Lemma 9] a sequence $x_{n}(\cdot)$ is weakly convergent in $C\left(I_{d}, E\right)$ to $x(\cdot)$ if 
and only if $x_{n}(t)$ tends weakly to $x(t)$, for each $t \in I_{d}$, so if $x_{n} \stackrel{\omega}{\longrightarrow} x$ in $C\left(I_{d}, E\right)$, then $k\left(t, s, x_{n}(s)\right) \stackrel{\omega}{\longrightarrow} k(t, s, x(s))$ in $E$ for $t \in I_{d}$ and by Theorem 2.12 (see our assumptions on $K_{1}$ ) we have

$$
\lim _{n \rightarrow \infty} \int_{0}^{t} k\left(z, s, x_{n}(s)\right) d s=\int_{0}^{t} k(z, s, x(s)) d s
$$

weakly in $E$, for each $t \in I_{d}$. Moreover, because $f$ is weakly-weakly sequentially continuous,

$$
f\left(t, x_{n}(t), \int_{0}^{t} k\left(t, s, x_{n}(s)\right) d s\right) \stackrel{\omega}{\longrightarrow} f\left(t, x(t), \int_{0}^{t} k(t, s, x(s)) d s\right)
$$

in $E$, for each $t \in I_{d}$. Thus, Theorem 2.12 (see our assumptions on $K$ ) implies $F\left(x_{n}\right)(t) \rightarrow F(x)(t)$ weakly in $E$, for each $t \in I_{d}$, so [26, Lemma 9] guarantees that $F\left(x_{n}\right) \rightarrow F(x)$ in $\left(C\left(I_{d}, E\right), \omega\right)$.

Suppose that $V \subset \widetilde{B}$ satisfies the condition $\bar{V}=\overline{\operatorname{conv}}(\{x\} \cup F(V))$. We shall prove that $V$ is relatively weakly compact and so (3.2) is satisfied. Since $V \subset \widetilde{B}$, $F(V) \subset K$. Then $V \subset \bar{V}=\overline{\operatorname{conv}}(\{x\} \cup F(V))$ is equicontinuous. By Lemma 1.1, $t \mapsto v(t)=\beta(V(t))$ is continuous on $I_{d}$.

For fixed $t \in I_{d}$ we divide the interval $[0, t]$ into $m$ parts: $0=t_{0}<t_{1}<\cdots<t_{m}$ $=t$, where $t_{i}=i t / m, i=0,1, \ldots, m$ and for fixed $z \in[0, t]$ we divide the interval $[0, z]$ into $m$ parts: $0=z_{0}<z_{1}<\cdots<z_{m}=z$, where $z_{j}=j z / m, j=0,1, \ldots, m$.

Let $V\left(\left[z_{j}, z_{j+1}\right]\right)=\left\{u(s) \mid u \in V, z_{j} \leq s \leq z_{j+1}\right\}, \quad j=0,1, \ldots, m-1 . \quad$ Вy Lemma 1.1 and the continuity of $v$ there exists $s_{j} \in I_{j}=\left[z_{j}, z_{j+1}\right]$ such that

$$
\beta\left(V\left(\left[z_{j}, z_{j+1}\right]\right)\right)=\sup \left\{\beta(V(s)) \mid z_{j} \leq s \leq z_{j+1}\right\}=: v\left(s_{j}\right) .
$$

By Theorem 2.11 and the properties of the HKP integral we have, for $x \in V$, that

$$
\begin{aligned}
F(x)(t)= & x_{0}+\sum_{i=0}^{m-1} \int_{t_{i}}^{t_{i+1}} f\left(z, x(z), \sum_{j=0}^{m-1} \int_{z_{j}}^{z_{j+1}} k(z, s, x(s)) d s\right) d z \\
& \in x_{0}+\sum_{i=0}^{m-1}\left(t_{i+1}-t_{i}\right) \overline{\operatorname{conv}} f\left(J_{i}, V\left(J_{i}\right), \sum_{j=0}^{m-1}\left(z_{j+1}-z_{j}\right) \overline{\operatorname{conv}} k\left(I_{j}, I_{j}, V\left(I_{j}\right)\right)\right),
\end{aligned}
$$

where $J_{i}=\left[t_{i}, t_{i+1}\right], i=0,1, \ldots, m-1$. 
Using (3.3), (3.4) and properties of the measure of weak noncompactness we obtain

$$
\begin{aligned}
\beta(F(V)(t)) \leq & \sum_{i=0}^{m-1}\left(t_{i+1}-t_{i}\right) \beta\left(f\left(J_{i}, V\left(J_{i}\right), \sum_{j=0}^{m-1}\left(z_{j+1}-z_{j}\right) \overline{\operatorname{conv}} k\left(I_{j}, I_{j}, V\left(I_{j}\right)\right)\right)\right) \\
\leq & \sum_{i=0}^{m-1}\left(t_{i+1}-t_{i}\right) \cdot c_{1} \cdot \beta\left(V\left(J_{i}\right)\right) \\
& +\sum_{i=0}^{m-1}\left(t_{i+1}-t_{i}\right) \cdot c_{2} \cdot \beta\left(\sum_{j=0}^{m-1}\left(z_{j+1}-z_{j}\right) \overline{\operatorname{conv}} k\left(I_{j}, I_{j}, V\left(I_{j}\right)\right)\right) \\
\leq & \sum_{i=0}^{m-1}\left(t_{i+1}-t_{i}\right) \cdot c_{1} \cdot \beta\left(V\left(I_{d}\right)\right) \\
& +\sum_{i=0}^{m-1}\left(t_{i+1}-t_{i}\right) \cdot c_{2} \cdot \sum_{j=0}^{m-1}\left(z_{j+1}-z_{j}\right) \beta\left(k\left(I_{j}, I_{j}, V\left(I_{j}\right)\right)\right. \\
\leq & \beta\left(V\left(I_{d}\right)\right) \cdot c_{1} \cdot d+\sum_{i=0}^{m-1}\left(t_{i+1}-t_{i}\right) \cdot c_{2} \cdot \sum_{j=0}^{m-1}\left(z_{j+1}-z_{j}\right) \cdot c_{3} \cdot \beta\left(V\left(I_{j}\right)\right) \\
\leq & \beta\left(V\left(I_{d}\right)\right) \cdot c_{1} \cdot d+\beta\left(V\left(I_{d}\right)\right) \cdot c_{2} \cdot c_{3} \cdot d^{2}=\beta\left(V\left(I_{d}\right)\right)\left(c_{1} \cdot d+c_{2} \cdot c_{3} \cdot d^{2}\right) .
\end{aligned}
$$

Because $\bar{V}=\overline{\operatorname{conv}}(\{x\} \cup F(V))$, then $\beta(V(t))=\beta(\overline{\operatorname{conv}}(\{x\} \cup F(V(t)))), \quad$ so $\beta(V(t)) \leq \beta\left(V\left(I_{d}\right)\right)\left(c_{1} \cdot d+c_{2} \cdot c_{3} \cdot d^{2}\right)$, for $t \in I_{d}$.

Using Lemma 1.1 we obtain

$$
\beta\left(V\left(I_{d}\right)\right) \leq \beta\left(V\left(I_{d}\right)\right)\left(c_{1} \cdot d+c_{2} \cdot c_{3} \cdot d^{2}\right) \beta\left(V\left(I_{d}\right)\right) \leq \beta\left(V\left(I_{d}\right)\right)\left(c_{1} \cdot d+c_{2} \cdot c_{3} \cdot d^{2}\right) .
$$

Since $0<d \cdot c_{1}+d^{2} \cdot c_{2} \cdot c_{3}<1$ we obtain $v(t)=\beta(V(t))=0$, for $t \in I_{d}$.

Using Arzela-Ascoli's theorem, we have that $V$ is relatively weakly compact.

By Theorem 3.2 the operator $F$ has a fixed point. This means that there exists a pseudo-solution of the problem (1.1).

THEOREM 3.4. Assume that for each uniformly $A C G^{*}$ function $x: I_{a} \rightarrow E$, the functions $k(\cdot, s, x(s)), \quad f\left(\cdot, x(\cdot), \int_{0}^{(\cdot)} k(\cdot, s, x(s)) d s\right)$ are HKP integrable and $k(t, s, \cdot), f(t, \cdot, \cdot)$ are weakly-weakly sequentially continuous functions. Suppose that there exists a constant $c>0$ and a continuous function $c_{1}: I_{a} \rightarrow R_{+}$such that

$$
\begin{aligned}
\beta(f(I, A, C)) & \leq c \cdot \beta(C) \quad \text { for each } A, C \subset B, I \subset I_{a}, \\
\beta(k(I, I, X)) & \leq \sup _{s \in I} c_{1}(s) \beta(X) \text { for each } X \subset B, I \subset I_{a},
\end{aligned}
$$

where

$$
\begin{aligned}
f(I, A, C) & =\left\{f\left(t, x_{1}, x_{2}\right) \mid\left(t, x_{1}, x_{2}\right) \in I \times A \times C\right\}, \\
k(I, I, X) & =\{k(t, s, x) \mid(t, s, x) \in I \times I \times X\}
\end{aligned}
$$

and $\beta$ denotes the measure of weak noncompactness of DeBlasi. 
Moreover, let $K$ and $K_{1}$ be equicontinuous and uniformly $A C G^{*}$ on $I_{a}$. Then there exists a pseudo-solution of the problem (1.1) on $I_{d}$, for some $0<d \leq a$.

PROOF. The first part of the proof is the same as in the proof of the previous theorem. It remains to show the relative weak compactness of $V$, where $V$ is defined in Theorem 3.3. In this case note that for $t \in I_{d}$ and $z_{j}$ as in Theorem 3.3

$$
\begin{aligned}
\beta(V(t)) \leq & \sum_{i=0}^{m-1}\left(t_{i+1}-t_{i}\right) \cdot c \cdot \beta\left(\sum_{j=0}^{m-1}\left(z_{j+1}-z_{j}\right) \overline{\operatorname{conv}} k\left(I_{j}, I_{j}, V\left(I_{j}\right)\right)\right) \\
\leq & \sum_{i=0}^{m-1}\left(t_{i+1}-t_{i}\right) \cdot c \cdot \sum_{j=0}^{m-1}\left(z_{j+1}-z_{j}\right) \beta\left(k\left(I_{j}, I_{j}, V\left(I_{j}\right)\right)\right) \\
\leq & \sum_{i=0}^{m-1}\left(t_{i+1}-t_{i}\right) \cdot c \cdot \sum_{j=0}^{m-1}\left(z_{j+1}-z_{j}\right) \sup _{s \in I_{j}} c_{1}(s) \beta\left(V\left(I_{j}\right)\right) \\
\leq & c \cdot d \cdot \sum_{j=0}^{m-1}\left(z_{j+1}-z_{j}\right) \cdot c_{1}\left(p_{j}\right) v\left(s_{j}\right) \\
= & c \cdot d\left(\sum_{j=0}^{m-1}\left(z_{j+1}-z_{j}\right) \cdot c_{1}\left(p_{j}\right) v\left(p_{j}\right)\right. \\
& \left.\quad+\sum_{j=0}^{m-1}\left(z_{j+1}-z_{j}\right)\left(c_{1}\left(p_{j}\right)\left(v\left(s_{j}\right)-v\left(p_{j}\right)\right)\right)\right)
\end{aligned}
$$

for some $p_{j} \in I_{j}$. Fix $\varepsilon>0$. From the continuity of $v$ we may choose $m$ large enough so that $v\left(s_{j}\right)-v\left(p_{j}\right)<\varepsilon$ and so

$$
\begin{aligned}
\beta(V(t)) & \leq c \cdot d\left(\sum_{j=0}^{m-1}\left(z_{j+1}-z_{j}\right) c_{1}\left(p_{j}\right) v\left(p_{j}\right)+\sum_{j=0}^{m-1} \frac{z}{m} c_{1}\left(p_{j}\right) \cdot \varepsilon\right) \\
& \leq c \cdot d\left(\sum_{j=0}^{m-1}\left(z_{j+1}-z_{j}\right) c_{1}\left(p_{j}\right) v\left(p_{j}\right)+z \cdot \varepsilon \cdot \max _{0 \leq k \leq m-1} c_{1}\left(p_{k}\right)\right) .
\end{aligned}
$$

Since $\varepsilon \rightarrow 0$ and $z \cdot \max _{0 \leq k \leq m-1} c_{1}\left(p_{k}\right)$ is bounded,

$$
z \cdot \varepsilon \cdot \max _{0 \leq k \leq m-1} c_{1}\left(p_{k}\right) \rightarrow 0
$$

Therefore

$$
v(t)=\beta(V(t)) \leq c \cdot d \cdot \int_{0}^{t} c_{1}(s) v(s) d s, \quad t \in[0, d] .
$$


Using the Gronwall's inequality we have that

$$
v(t)=\beta(V(t))=0 \quad \text { for } t \in[0, d] .
$$

Using Arzela-Ascoli's theorem we deduce that $V$ is relatively weakly compact.

By Theorem 3.2 the operator $F$ has a fixed point. This means that there exists a pseudo-solution of the problem (1.1).

\section{Compactness and connectedness}

In this section we show that the set $S$ of all solutions of the problem (1.1) on $I_{d}$ is compact and connected in $\left(C\left(I_{d}, E\right), \omega\right)$.

THEOREM 4.1. Under the assumptions of Theorem 3.3 a set $S$ of all pseudo-solutions of the problem (1.1) on $I_{d}$ is weakly compact and connected in $\left(C\left(I_{d}, E\right), \omega\right)$.

PROOF. Let $S$ be a set of all solutions of the problem (1.1) on $I_{d}$. As $S=F(S)$, by repeating the above argument, with $V=S$ one can show that $S$ is relatively weakly compact in $\left(C\left(I_{d}, E\right), \omega\right)$. Since $F$ is weakly continuous on $\overline{S\left(I_{d}\right)^{\omega}}, S$ is weakly closed and consequently weakly compact.

Now we prove that $S$ is connected. For any $\eta>0$, denote by $S_{\eta}$, the set of all functions $u: I_{d} \rightarrow E$ satisfying the following conditions:

(i) $u(0)=x_{0}, u \in \widetilde{B}$,

(ii) $\sup _{t \in I_{d}}\left\|u(t)-x_{0}-\int_{0}^{t} f\left(z, x(z), \int_{0}^{z} k(z, s, x(s)) d s\right) d z\right\|<\eta$.

The set $S_{\eta}$ is nonempty as $S \subset S_{\eta}$.

Let $\eta^{*}<\eta$. By the equicontinuity of $K$ we can choose $\rho$ such that

$$
\left\|\int_{J} f\left(z, x(z), \int_{0}^{z} k(z, s, x(s)) d s\right) d z\right\| \leq \eta^{*}<\eta,
$$

for any $x \in\left(C\left(I_{d}, E\right), \omega\right), J \subset I_{d}$ and $|J|<\rho$.

For any $\varepsilon \in(0, d)$, let $v(\cdot, \varepsilon): I_{d} \rightarrow E$ be defined by the formula:

$$
v(t, \varepsilon)= \begin{cases}x_{0} & \text { for } 0 \leq t \leq \varepsilon \\ x_{0}+\int_{0}^{t-\varepsilon} f\left(z, x(z), \int_{0}^{z} k(z, s, v(s, \varepsilon)) d s\right) d z & \text { for } \varepsilon<t \leq d .\end{cases}
$$

Clearly $v(\cdot, \varepsilon)$ satisfies (i) above. Furthermore, for $0<\varepsilon \leq \min (\rho, d)=l$

$$
\begin{aligned}
& \left\|v(t, \varepsilon)-x_{0}-\int_{0}^{t} f\left(z, x(z), \int_{0}^{z} k(z, s, v(s, \varepsilon)) d s\right) d z\right\|
\end{aligned}
$$

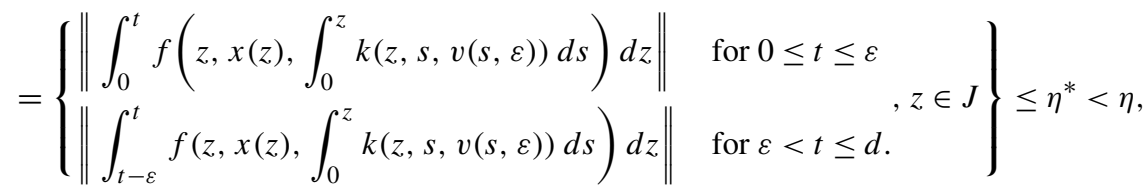

thus $v(\cdot, \varepsilon)$ satisfies (ii) above. 
Now, we prove that $S_{\eta}$ is connected. Let us define

$$
v_{\varepsilon}(t)= \begin{cases}x_{0}, & 0 \leq t \leq \varepsilon \\ F\left(v_{\varepsilon}\right)(t-\varepsilon), & \varepsilon<t \leq d\end{cases}
$$

where $v_{\varepsilon}=v(\cdot, \varepsilon)$. We show that the mapping $\varepsilon \rightarrow v_{\varepsilon}(\cdot)$ is sequentially continuous from $(0, d)$ into $\left(C\left(I_{d}, E\right), \omega\right)$.

Let $0<\varepsilon<\delta \leq d$ (when $\delta \leq \varepsilon$ the argument is similar). Let $x^{*} \in E^{*}$ be such that $\left\|x^{*}\right\| \leq 1$. Now by the definition of $v_{\varepsilon}(t)$, for $t \in[0, \varepsilon]$

$$
\left|x^{*}\left(v_{\varepsilon}(t)-v_{\delta}(t)\right)\right|=0 .
$$

Next, if $t \in(\varepsilon, \delta]$,

$$
\begin{aligned}
\left|x^{*}\left(v_{\varepsilon}(t)-v_{\delta}(t)\right)\right|=\mid x^{*}\left[\int_{0}^{t-\varepsilon} f\left(z, x(z), \int_{0}^{z} k\left(z, s, v_{\varepsilon}(s)\right) d s\right) d z\right. \\
\left.\quad-\int_{0}^{t-\delta} f\left(z, x(z), \int_{0}^{z} k\left(z, s, v_{\delta}(s)\right) d s\right) d z\right] \mid \\
=\left|x^{*} \int_{t-\delta}^{t-\varepsilon} f\left(z, x(z), \int_{0}^{z} k\left(z, s, v_{\varepsilon}(s)\right) d s\right) d z\right| \\
=\left\|x^{*}\right\|\left\|\int_{t-\delta}^{t-\varepsilon} f\left(z, x(z), \int_{0}^{z} k\left(z, s, v_{\varepsilon}(s)\right) d s\right) d z\right\| \\
\leq\left\|\int_{t-\delta}^{t-\varepsilon} f\left(z, x(z), \int_{0}^{z} k\left(z, s, v_{\varepsilon}(s)\right) d s\right) d z\right\|
\end{aligned}
$$

Consequently

$$
\left|x^{*}\left(v_{\varepsilon}(t)-v_{\delta}(t)\right)\right| \leq\left\|\int_{t-\delta}^{t-\varepsilon} f\left(z, x(z), \int_{0}^{z} k\left(z, s, v_{\varepsilon}(s)\right) d s\right) d z\right\|:=A_{\delta} .
$$

Since $K$ is equicontinuous, note that if $\delta \rightarrow \varepsilon$, then $A_{\delta} \rightarrow 0$.

Now, for $t \in(\delta, 2 \delta]$, we have

$$
\begin{aligned}
\left|x^{*}\left(v_{\varepsilon}(t)-v_{\delta}(t)\right)\right| & \mid x^{*}\left[\int_{0}^{t-\varepsilon} f\left(z, x(z), \int_{0}^{z} k\left(z, s, v_{\varepsilon}(s)\right) d s\right) d z\right. \\
& \left.-\int_{0}^{t-\delta} f\left(z, x(z), \int_{0}^{z} k\left(z, s, v_{\delta}(s)\right) d s\right) d z\right] \mid \\
= & \left|x^{*}\left(F\left(v_{\varepsilon}\right)(t-\varepsilon)-F\left(v_{\delta}\right)(t-\delta)\right)\right| \\
= & \left|x^{*}\left[F\left(v_{\varepsilon}\right)(t-\varepsilon)-F\left(v_{\varepsilon}\right)(t-\delta)+F\left(v_{\varepsilon}\right)(t-\delta)-F\left(v_{\delta}\right)(t-\delta)\right]\right| \\
\leq & \left|x^{*}\left(F\left(v_{\varepsilon}\right)(t-\varepsilon)-F\left(v_{\varepsilon}\right)(t-\delta)\right)\right|+\left|x^{*}\left(F\left(v_{\varepsilon}\right)(t-\delta)-F\left(v_{\delta}\right)(t-\delta)\right)\right| \\
\leq & \left\|x^{*}\right\|\left\|F\left(v_{\varepsilon}\right)(t-\varepsilon)-F\left(v_{\varepsilon}\right)(t-\delta)\right\|+\left\|x^{*}\right\|\left\|F\left(v_{\varepsilon}\right)(t-\delta)-F\left(v_{\delta}\right)(t-\delta)\right\| .
\end{aligned}
$$


Let $\left(\delta_{n}\right)$ be a sequence such that $\delta_{n} \rightarrow \varepsilon\left(\varepsilon \leq \delta_{n}\right)$. By (4.1) and (4.2) it follows that $v_{\delta_{n}}(t)$ converges to $v_{\varepsilon}(t)$ weakly uniformly for $t \in[0, \delta]$. Thus, $F\left(v_{\delta_{n}}\right)(t) \rightarrow F\left(v_{\varepsilon}\right)(t)$ weakly on $[0, \delta]$. Now, by (4.3) $v_{\delta_{n}}(t)$ tends to $v_{\varepsilon}(t)$ weakly for each $t \in[0,2 \delta]$.

By repeating the above argument and using induction, we obtain that the map $\varepsilon \rightarrow v_{\varepsilon}(\cdot)$ from $(0, d)$ into $\left(C\left(I_{d}, E\right), \omega\right)$ is sequentially continuous [26, Lemma 1.9]. Therefore, by Lemma 1.2 the set $V=\left\{v_{\varepsilon}(\cdot) \mid 0<\varepsilon<d\right\}$ is connected in $\left(C\left(I_{d}, E\right), \omega\right)$ (because the interval $[0, d]$ is connected).

Let $x \in S_{\eta}$. Let us choose $\varepsilon>0$ such that $0<\varepsilon<d$ and

$$
\begin{aligned}
& \sup _{t \in I_{d}}\left\|x(t)-x_{0}-\int_{0}^{t} f\left(z, x(z), \int_{0}^{z} k(z, s, x(s)) d s\right) d z t\right\| \\
& \quad+\left\|\int_{I_{\varepsilon}} f\left(z, x(z), \int_{0}^{z} k(z, s, x(s)) d s\right) d z\right\|<\eta
\end{aligned}
$$

For any $p, 0 \leq p \leq d$, let $y(\cdot, p): I_{d} \rightarrow E$ be defined by the formula:

$$
\left[y(t, p)=\left\{\begin{array}{ll}
x(t), & \text { for } 0 \leq t \leq p \\
x(p)+\frac{x_{0}-x(p)}{\varepsilon}(t-p), & \text { for } p<t \leq \min (d, p+\varepsilon) \\
x_{0}+\int_{p}^{t-\varepsilon} f\left(z, x(z), \int_{0}^{z} k(z, s, y(s, p)) d s\right) d z, & \text { for } \min (d, p+\varepsilon)<t<d
\end{array}\right\}\right.
$$
$y(t, 0)=v(t, \varepsilon)$.

By repeating the above argument with $y(\cdot, p)$ in the place of $v(\cdot, \varepsilon)$ one can show that $y(\cdot, p) \in S_{\eta}$, for each $p \in[0, d]$ and the mapping $p \rightarrow y(\cdot, p)$ from $I_{d}$ into $\left(C\left(I_{d}, E\right), \omega\right)$ is sequentially continuous (for more details see [22, 30]).

Consequently, by Lemma 1.2 the set $T_{x}=\{y(\cdot, p) \mid 0 \leq p \leq d\}$ is connected in $\left(C\left(I_{d}, E\right), \omega\right)$.

Now since $y(\cdot, 0)=v(\cdot, \varepsilon) \in V \cap T_{x}$, the set $V \cup T_{x}$ is connected and therefore the set $W=\bigcup_{x \in S_{\eta}} T_{x} \cup V$ is connected in $\left(C\left(I_{d}, E\right), \omega\right)$.

Moreover, $S_{\eta} \subset W$, because $x=y(\cdot, p) \in T_{x}$, for each $x \in S_{\eta}$. On the other hand $W \subset S_{\eta}$, since $T_{x} \subset S_{\eta}$ and $V \subset S_{\eta}$. Thus, $S_{\eta}=W$ is a connected subset of $\left(C\left(I_{d}, E\right), \omega\right)$.

Now, suppose that the set $S$ is not connected. As $S$ is weakly compact, there exists nonempty weakly compact sets $W_{1}$ and $W_{2}$, such that $S=W_{1} \cup W_{2}$ and $S=W_{1} \cup W_{2}$. Consequently, there exist two disjoint weakly open sets $U_{1}, U_{2}$, such that $W_{1} \cap W_{2}$ $=\emptyset, W_{2} \subset U_{2}$. Suppose that, for every $n \in N$, there exists $u_{n} \in V_{n} \backslash U$, where $V_{n}=\bar{S}_{1 / n}^{\omega}$ and $U=U_{1} \cup U_{2}$. Note that $V_{n}$ is a decreasing sequence of nonempty weakly compact connected subsets of $\left(C\left(I_{d}, E\right), \omega\right)$.

Let $H=\overline{\left\{u_{n} \mid n \in N\right\}^{\omega}}$. Note that $u_{n}-F\left(u_{n}\right) \rightarrow 0$ in $\left(C\left(I_{d}, E\right), \omega\right)$ as $n \rightarrow \infty$ and $H(t) \subset\left\{u_{n}(t)-F\left(u_{n}\right)(t) \mid u_{n} \in H\right\}+F(H)(t)$. By repeating the argument from the proof of Theorem 3.3, one can show that there exists $u_{0} \in H$ such that $u_{0}=F\left(u_{0}\right)$, that is, $u_{0} \in S$.

Now since $u_{n} \in V_{n} \backslash U$ and $U$ is weakly open we have $u_{0} \notin U$. This contradicts $u_{0} \in S \subset U$. 
Therefore, there exists $m \in N$ such that $V_{m} \subset U=U_{1} \cup U_{2}, U_{1} \cap U_{2}=\emptyset$. Now since $S \subset V_{m}$, we have that $U_{1} \cap V_{m} \neq \emptyset \neq U_{2} \cap V_{m}$. Thus, $V_{m}$ is not connected, a contradiction with the connectedness of each $V_{n}$. Consequently, $S$ is connected in $\left(C\left(I_{d}, E\right), \omega\right)$.

\section{References}

[1] R. P. Agarwal, M. Meehan and D. O'Regan, 'Positive solutions of singular integral equations-a survey', Dynam. Systems Appl. 14(1) (2005), 1-37.

[2] - Nonlinear Integral Equations and Inclusions (Nova Science Publishers, Hauppauge, NY, 2001).

[3] R. P. Agarwal and D. O'Regan, 'Existence results for singular integral equations of Fredholm type', Appl. Math. Lett. 13(2) (2000), 27-34.

[4] Z. Artstein, 'Topological dynamics of ordinary differential equations and Kurzweil equations', J. Differential Equations 23 (1977), 224-243.

[5] J. Banaś and J. Rivero, 'On measures of weak noncompactness', Ann. Mat. Pura Appl. 125 (1987), 213-224.

[6] F. S. DeBlasi, 'On a property of the unit sphere in a Banach space', Bull. Math. Soc. Sci. Math. R. S. Roumanie 21 (1977), 259-262.

[7] S. S. Cao, 'The Henstock integral for Banach valued functions', SEA Bull. Math. 16 (1992), 36-40.

[8] T. S. Chew, 'On Kurzweil generalized ordinary differential equations', J. Differential Equations 76 (1988), 286-293.

[9] T. S. Chew and F. Flordeliza, 'On $x^{\prime}=f(t, x)$ and Henstock-Kurzweil integrals', Differential Integral Equations 4 (1991), 861-868.

[10] M. Cichoń, 'Convergence theorems for the Henstock-Kurzweil-Pettis integral', Acta Math. Hungarica 92 (2001), 75-82.

[11] M. Cichoń and I. Kubiaczyk, 'On the set of solutions of the Cauchy problem in Banach spaces', Arch. Math. 63 (1994), 251-257.

[12] M. Cichoń, I. Kubiaczyk and A. Sikorska, 'Henstock-Kurzweil and Henstock-Kurzweil-Pettis integrals and some existence theorems', in: Proc. ISCM Herlany 1999. 2000, pp. 53-56.

[13] - 'The Henstock-Kurzweil-Pettis integrals and existence theorems for the Cauchy problem', Czechoslovak Math. J. 54 (2004), 279-289.

[14] F. Cramer, V. Lakshmikantham and A. R. Mitchell, 'On the existence of weak solution of differential equations in nonreflexive Banach spaces', Nonlinear. Anal., TMA 2 (1978), 169-177.

[15] R. F. Geitz, 'Pettis integration', Proc. Amer. Math. Soc. 82 (1991), 81-86.

[16] R. A. Gordon, 'The McShane integral of Banach-valued functions', Illinois J. Math. 34 (1990), 557-567.

[17] - The Integrals of Lebesgue, Denjoy, Perron and Henstock (American Mathematical Society, Providence, RI, 1994).

[18] _ - 'Riemann integration in Banach spaces', Rocky Mountain J. Math. 21 (1991), 923-949.

[19] R. Henstock, The General Theory of Integration, Oxford Math. Monographs (Clarendon, Oxford, 1991).

[20] J. Januszewski, 'On the existence of continuous solutions of nonlinear integral equations in Banach spaces', Comment. Math. 30 (1990), 85-92.

[21] S. Krzyśka, 'On the existence of continuous solutions of Urysohn and Volterra integral equations in Banach spaces', Demonstratio Math. 2 (1995), 353-359.

[22] I. Kubiaczyk, 'Kneser type theorems for ordinary differential equations in Banach spaces', J. Differential Equations 45 (1982), 139-146.

[23] I. Kubiaczyk and A. Sikorska, 'Differential equations in Banach spaces and Henstock-Kurzweil integrals', Discuss. Math. Differ. Incl. 19 (1999), 35-43. 
[24] P. Y. Lee, Lanzhou Lectures on Henstock Integration, Ser. Real. Anal., 2 (World Scientific, Singapore, 1989).

[25] M. Meehan and D. O'Regan, 'Existence theory for nonlinear Volterra integrodifferential and integral equations', Nonlinear Anal. TMA 31(3/4) (1998), 317-341.

[26] A. R. Mitchell and Ch. Smith, 'An existence theorem for weak solutions of differential equations in Banach spaces', in: Nonlinear Equations in Abstract Spaces (Academic Press, New York, 1978), pp. 387-404.

[27] D. O’Regan, 'Existence results for nonlinear integral equations', J. Math. Anal. Appl. 192(3) (1995), 705-726.

[28] D. O'Regan and M. Meehan, Existence Theory for Integral and Integrodifferential Equations, Mathematics and Its Applications, 445 (Kluwer Academic, Dordrecht, 1998).

[29] B. J. Pettis, 'On integration in vector spaces', Trans. Amer. Math. Soc. 44 (1938), 277-304.

[30] S. Szufla, 'Kneser's theorem for weak solutions for ordinary differential equations in reflexive Banach spaces', Bull. Polish Acad. Sci. Math. 26 (1978), 407-413.

RAVI P. AGARWAL, Department of Mathematical Sciences, Florida Institute of Technology, Melbourne, FL 32901, USA e-mail: agarwal@fit.edu

DONAL O'REGAN, Department of Mathematics, National University of Ireland, Galway, Ireland

e-mail: donal.oregan@nuigalway.ie

ANETA SIKORSKA-NOWAK, Faculty of Mathematics and Computer Science, Adam Mickiewicz University, Poznań, Poland

e-mail: anetas@amu.edu.pl 